\title{
DIMENSION STRUCTURES
}

\author{
ATTILA LOSONCZI
}

Abstract. We are going to introduce a new algebraic, analytic structure that is a kind of generalization of the Hausdorff dimension and measure. We give many examples and study the basic properties and relations of such systems.

Mathematics subject classification (2010): 28A78.

Keywords and phrases: Hausdorff dimension and measure.

\section{REFERENCES}

[1] P. Billingsley, Probability and Measure, John Wiley \& Sons, vol. 939 (2012).

[2] R. Engelking, Dimension theory, North-Holland Publishing Company (1978).

[3] R. Engelking, General topology, Sigma Series in Pure Mathematics, vol. 6. (1989).

[4] K. FALCONER, Fractal geometry: mathematical foundations and applications, John Wiley \& Sons (2004).

[5] F. HausdorfF, Dimension and outer measure, Mathematische Annalen 79.1 (1918), 157-179.

[6] A. LosoncZI, Measures by means, means by measures, arXiv preprint.

[7] B. B. Mandelbrot, R. Pignoni, The fractal geometry of nature, New York: WH freeman Vol. 173 (1983).

[8] S. Roman, Lattices and ordered sets, Springer Science \& Business Media (2008). 